\title{
Fitorremediação de solos contaminados com sulfentrazone em função da inoculação de bactérias simbióticas em Crotalaria juncea
}

\begin{abstract}
A simbiose entre plantas fitorremediadoras e microrganismos com atividade enzimática metabolizadora de herbicidas pode ser uma alternativa eficiente para remediação de solos contaminados com sulfentrazone. Objetivou-se avaliar a Crotalaria juncea (crotalária) inoculada com Bradyrhizobium sp. na fitorremediação do sulfentrazone e seus efeitos na biomassa microbiana do solo. O experimento foi conduzido em casa-de-vegetação da Universidade Federal do Espírito Santo (UFES), testando-se quatro doses de sulfentrazone (0, 400, 800 e $1200 \mathrm{~g}$ i.a. ha-1) na presença ou ausência da inoculação com Bradyrhizobium sp. BR2003 (SEMIA 6156). Após 65 dias de cultivo, as plantas foram cortadas, o solo foi coletado para as análises da biomassa microbiana e o bioensaio com o milheto. A inoculação com Bradyrhizobium sp. proporcionou melhor desenvolvimento em altura das plantas de crotalária, entretanto, o sistema radicular não foi influenciado pela adição do microrganismo. Os indicadores biológicos do solo mostraram que a inoculação com Bradyrhizobium sp. influenciou de maneira negativa na atividade microbiana dos solos. A crotalária apresentou boa tolerância aos níveis de sulfentrazone no solo, independentemente do inoculante. Os resultados sugerem que a inoculação da crotalária com Bradyrhizobium sp. não mostrou benefício na eficácia da remediação dos sulfentrazone, tendo a crotalaria melhor desempenho com os microrganismos nativos do solo.
\end{abstract}

Palavras-chave: Leguminosas; Microrganismos simbióticos; Descontaminação ambiental; Herbicidas.

\section{Phytorremediation of soil contaminated with sulfentrazone in function of the inoculation of symbotic bacteria in Crotalaria juncea}

\begin{abstract}
The symbiosis between phytoremediation plants and microorganisms with enzymatic activity that metabolizes herbicides can be an efficient alternative for the remediation of soils contaminated with sulfentrazone. The objective was to evaluate Crotalaria juncea (crotalária) inoculated with Bradyrhizobium sp. in the phytoremediation of sulfentrazone and its effects on soil microbial biomass. The experiment was conducted in a greenhouse at the Universidade Federal do Espírito Santo (UFES), testing four doses of sulfentrazone $(0,400,800$ and $1200 \mathrm{~g}$ i.a. ha-1) in the presence or absence of inoculation with Bradyrhizobium sp. BR2003 (SEMIA 6156). After 65 days of cultivation, the plants were cut, the soil was collected for the analysis of microbial biomass and the bioassay with millet. Inoculation with Bradyrhizobium sp. provided better development in height of the crotalaria plants, however, the root system was not influenced by the addition of the microorganism. The biological indicators of the soil showed that inoculation with Bradyrhizobium sp. negatively influenced soil microbial activity. The crotalaria showed good tolerance to the levels of sulfentrazone in the soil, regardless of the inoculant. The results suggest that inoculation of crotalária with Bradyrhizobium $\mathrm{sp}$. showed no benefit in the efficacy of sulfentrazone remediation, with the crotalaria having a better performance with native soil microorganisms.
\end{abstract}

Keywords: Legumes; Symbiotic microorganisms; Environmental decontamination; Herbicides.

Topic: Microbiologia Agrícola e Ambiental

Reviewed anonymously in the process of blind peer
Received: 08/06/2021

Approved: 24/06/2021
Kamila Cabral Mielke (iD

Universidade Federal de Viçosa, Brasil http://lattes.cnpq.br/9582543119922983

http://orcid.org/0000-0001-9576-9928

kamila.mielke@ufv.br

Rafaela Ramos Bertuani

Universidade Federal do Espírito Santo, Brasil http://lattes.cnpq.br/1638113412421210

rafaelabertuani22@gmail.com

Ana Flávia Souza Laube (it)

Universidade Federal de Viçosa, Brasil http://lattes.cnpq.br/7385111214626928 http://orcid.org/0000-0001-5938-1424 anaf.laube@gmail.com

\begin{abstract}
Maura Gabriela da Silva Brochado (iD Universidade Federal de Viçosa, Brasil http://lattes.cnpq.br/1509312557137003 http://orcid.org/0000-0003-1981-121X maurabrochado@gmail.com
\end{abstract}

Ana Carolina Melo Ribeiro ic

Universidade Estadual do Norte Fluminense Darcy Ribeiro, Brasil http://lattes.cnpq.br/0917987913594738 http://orcid.org/0000-0003-0891-2445 carolm.ribeiro95@gmil.com

Dilma Francisca de Paula

Universidade Federal de Viçosa, Brasil http://lattes.cnpq.br/5000058539625268 dilmafdepaula@gmail.com
Fábio Ribeiro Pires (iD)

Universidade Federal do Espírito Santo, Brasil http://lattes.cnpq.br/5930035056050041

http://orcid.org/0000-0001-6534-3475 pires.fr@gmail.com

\section{Rafael D'Angeri}

Universidade Federal de Viçosa, Brasil http://lattes.cnpq.br/2623230615305828 rafael.dangeri@ufv.br

DOI: 10.6008/CBPC2179-6858.2021.006.0012

Referencing this:

MIELKE, K. C.; BERTUANI, R. R.; LAUBE, A. F. S.; BROCHADO, M. G. S.; RIBEIRO, A. C. M.; PAULA, D. F.; PIRES, F. R.; D'ANGIERI, R..

Fitorremediação de solos contaminados com sulfentrazone em função da inoculação de bactérias simbióticas em Crotalaria juncea. Revista Ibero Americana de Ciências Ambientais, v.12, n.6, p.140-151, 2021. DOI: http://doi.org/10.6008/CBPC2179-6858.2021.006.0012 


\section{INTRODUÇÃO}

Os agrotóxicos são amplamente utilizados nas atividades agrícolas atuais, possibilitando o cultivo de extensas áreas. O uso impróprio desses produtos químicos já causou ao longo dos anos, considerável poluição ambiental e sua exposição contínua pode proporcionar risco para organismos não-alvo, como plantas, animais e a população humana (PELCASTRE et al., 2013). Os herbicidas desempenham um papel fundamental na produção agrícola, entretanto, podem contaminar o meio ambiente através da lixiviação para águas subterrâneas.

Alguns herbicidas apresentam elevada persistência no ambiente, o que implica no efeito residual do composto no solo, impedindo o desenvolvimento de plantas daninhas durante determinado período (DAN et al., 2010). Entretanto, herbicidas com bioatividade prolongada tornam-se indesejáveis, provocando intoxicações em plantas sensíveis, cultivadas em sucessão ou rotação de culturas (OLIVEIRA et al., 2014). Tais compostos podem afetar a atividade microbiana (IMFELD et al., 2012) e contaminar águas subterrâneas e nascentes (SANTOS et al., 2015). O sulfentrazone (N-[2,4-dicloro-5-[4-(difluorometil)-4,5-dihidro-3metil-5oxo-1H-1,2,4-triazol -1-il] metanosulfonamida) é um herbicida pré-emergente, pertencente ao grupo das aril triazolinonas, que apresenta atividade residual prolongada entre 6 e 24 meses, dependendo do tipo de solo e condições ambientais (MARTINEZ et al., 2008; MONQUEIRO et al., 2010).

Estudos para o desenvolvimento de técnicas de remediação/mitigação dos danos causados pela presença de herbicidas no solo têm sido intensificados (SANTOS et al., 2018; MELO et al., 2019; SOUZA et al., 2017). Uma promissora técnica de descontaminação que utiliza recursos naturais para recuperar um ambiente contaminado e tóxico a uma condição segura é a biorremediação (KANG, 2014). O uso de plantas na remediação é chamado de fitorremediação, baseada na ação combinada de plantas e comunidades microbianas associadas para degradar, remover, transformar ou imobilizar compostos tóxicos (TRUU et al., 2015).

Cientistas ambientais têm estudado o potencial da simbiose de leguminosas e micróbios para remediar os solos contaminados com poluentes orgânicos (KAWASAKI et al., 2011). As leguminosas são muito importantes tanto em termos ecológicos como agrícolas, porque são tolerantes a diferentes condições climáticas (ZAHRAN, 1999). Os nódulos radiculares são importantes para a fixação biológica de nitrogênio do sistema simbiótico, sendo fisiologicamente e morfologicamente distintos de outros órgãos da planta. Devido às propriedades de fixação de nitrogênio, as leguminosas aumentam a acumulação de nitrogênio e matéria orgânica, equilibram a relação carbono/nitrogênio e aumentam a quantidade de microrganismos de rizosfera (GAGE, 2004).

Estudos demonstraram que a associação de plantas e microrganismos, como fungos micorrízicos pode ter significante efeito na absorção de urânio pelas plantas (RUFYIKIRI et al., 2003). Shaw et al. (2004) mostraram que a mineralização do 2,4-D foi aumentada na rizosfera de Trifolium pratense devido ao aumento do tamanho populacional de degradadores 2,4-D na rizosfera. Estudando o emprego do consórcio de microrganismos, a partir de amostras de petróleo, Costa et al. (2007) conseguiram observar a eficiência 
desse processo na descontaminação de áreas contaminadas. Logo, objetivou-se avaliar a Crotalaria juncea inoculada com Bradyrhizobium sp. na fitorremediação do sulfentrazone e seus efeitos na biomassa microbiana do solo.

\section{MATERIAIS E MÉTODOS}

O experimento foi conduzido, entre 08/2019 e 07/2020, em casa-de-vegetação localizada na Universidade Federal do Espírito Santo (UFES), em São Mateus-ES. O solo utilizado foi classificado como Argissolo Amarelo Distrófico de textura média (EMBRAPA, 2018) característico da região, coletado na Fazenda Experimental localizada no CEUNES/UFES, em área sem histórico de aplicação de herbicidas, na profundidade de 0,0-20,0 cm. O solo possuía 106, 10 e $884 \mathrm{~g} \mathrm{~kg}^{-1}$ de argila, silte e areia, respectivamente, com as seguintes características químicas: $\mathrm{pH} 4,7$; matéria orgânica $21,0 \mathrm{~g} \mathrm{~kg}^{-1}$; P e K 1,2 e $16 \mathrm{mg} \mathrm{dm}^{3}$, respectivamente; $\mathrm{Ca}, \mathrm{Mg}$, $\mathrm{Al}$ e $\mathrm{H}+\mathrm{Al}, \mathrm{CTC}_{\mathrm{e}}$ e $\mathrm{CTC}_{\mathrm{pH}=7,0} 0,4,0,2,1,0,5,8,1,7$ e $6,5 \mathrm{cmolc} \mathrm{dm}^{3}$, respectivamente; e saturação por bases $10,3 \%$.

O experimento foi conduzido em blocos casualizados com quatro repetições em esquema fatorial 4x2. O primeiro fator foi composto por quatro doses de sulfentrazone $\left(0,400,800\right.$ e $\left.1200 \mathrm{~g}^{\text {i.a. }} \mathrm{ha}^{-1}\right)$ e o segundo fator foi constituído pela presença ou ausência da inoculação com Bradyrhizobium sp, aplicado à espécie fitorremediadora crotalária (Crotalaria juncea).

O solo foi submetido à esterilização em autoclave vertical, à pressão de 1,0 atm e temperatura de $120^{\circ} \mathrm{C}$ por 40 minutos. Cada unidade experimental correspondeu a um vaso de polietileno com 15,0 kg de solo, revestidos com sacos plásticos para impedir a perda de herbicida juntamente com o solo. A aplicação do sulfentrazone foi realizada, sobre o solo presente no vaso, utilizando-se pulverizador pressurizado com $\mathrm{CO}_{2}$, com duas pontas espaçadas por $0,5 \mathrm{~m}$, bico tipo leque XR11002VP, com volume de calda de $147 \mathrm{~L} \mathrm{ha}^{-1}$, 24 horas antes do plantio.

O inoculante foi o sólido turfoso para crotalária, Bradyrhizobium sp. BR2003 (SEMIA 6156). Para inoculação das sementes as sementes foram desinfestadas em álcool 70\% por 30s e em seguida imergidas em solução de hipoclorito de sódio 1\% por 3 min. Após a desinfecção, as sementes foram lavadas duas vezes sucessivas em água destilada. Preparou-se $20 \mathrm{~mL}$ de solução açucarada $10 \%$ para umedecimento das sementes e foram adicionados $25 \mathrm{~g}$ do inoculante sobre as sementes, homogeneizando-se até completa cobertura. As sementes inoculadas foram secas ao ar por 30 minutos. Foram semeadas 10 sementes de crotalária por vaso, na profundidade de 5,0 cm. Após 15 dias de semeadura, realizou-se o desbaste, deixandose duas plantas em cada unidade experimental. As irrigações foram realizadas três vezes ao dia, a fim de manter a umidade do solo a $60 \%$ da capacidade de campo.

Foi avaliada altura de plantas $(\mathrm{cm})$ aos 65 dias após a semeadura (DAS), tomando-se como base para medição o meristema apical. As plantas foram cortadas na altura do coleto, sendo a massa do material vegetal imediatamente registrada, denominada de biomassa fresca da parte aérea. As raízes foram cuidadosamente retiradas dos vasos, intactas e foram retirados todos os nódulos presentes para contagem do número de nódulos total. A massa seca de nódulos $\left(\mathrm{g} \mathrm{plantas}^{-1}\right.$ ) foi avaliada por meio da secagem dos 
nódulos em estufa de circulação forçada de $\operatorname{ar}\left(70 \pm 2^{\circ} \mathrm{C}\right)$, seguida do registro da massa em balança analítica (precisão 0,1 mg) até massa constante. As raízes foram então lavadas e pesadas para obtenção da biomassa fresca. Posteriormente, o material vegetal (parte aérea e raiz) foi colocado em estufa de circulação forçada de $\operatorname{ar}\left(70 \pm 2^{\circ} \mathrm{C}\right)$ até massa constante.

O milheto (Pennisetum glaucum) foi utilizado por apresentar elevada suscetibilidade ao sulfentrazone, conforme reportado por (BELO et al., 2016; DAN et al., 2011). Vasos plásticos com fundo selado foram preenchidos com $0,5 \mathrm{~kg}$ de solo de cada tratamento após o cultivo da crotalaria. Posteriormente, foram semeadas 15 sementes do milheto (var. ADR7010), mantendo-se seis plântulas por vaso após o desbaste. Aos 30 DAS foi avaliada altura de plantas $(\mathrm{cm})$ tendo como referência o meristema apical. A intoxicação visual foi avaliada utilizando-se escala percentual, onde 0 (zero) significa ausência de sintomas (redução da altura das plantas, clorose e necrose das folhas), e $100 \%$ morte de todas as plantas. No mesmo dia, determinou-se a massa seca da parte aérea, em comparação às plantas do tratamento controle, após secagem até peso constante, a $70 \pm 2 \circ \mathrm{C}$.

$\mathrm{Na}$ quantificação da frequência respiratória da microbiota do solo foi utilizado o método respirométrico de avaliação do $\mathrm{C}-\mathrm{CO}_{2}$ evoluído. Amostra de $150 \mathrm{~g}$ de solo após o cultivo com a crotalaria, foi peneirada (malha 2,00 $\mathrm{mm}$ ) e umedecida ( $70 \%$ da capacidade de campo) e incubadas durante 40 dias em frascos hermeticamente fechados em temperatura ambiente. As avaliações ocorreram 1, 5, 10, 15, 20, 25, 30, 35 e 40 dias após o início da incubação. $\mathrm{O} \mathrm{C}-\mathrm{CO}_{2}$ liberado do solo foi carreado por fluxo contínuo de ar (isento de $\mathrm{CO}_{2}$ ) até outro frasco contendo $10 \mathrm{~mL}$ de solução de $\mathrm{NaOH} 0,5 \mathrm{~mol} \mathrm{~L}^{-1}$. Para sua liberação foram

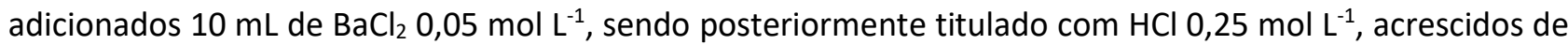
três gotas do indicador fenolftaleína a 1\% (STOTZKY, 1965; CURL et al., 1972).

Após 40 dias, $17 \mathrm{~g}$ de solo de cada frasco foram pesados para determinação do carbono da biomassa microbiana (CBM) conforme Vance et al. (1987), modificado por Islam et al. (1998). A partir dos valores obtidos de $\mathrm{C}-\mathrm{CO}_{2}$ e $\mathrm{CBM}$ o quociente metabólico $\left(q \mathrm{CO}_{2}\right)$ foi calculado dividindo-se o $\mathrm{C}-\mathrm{CO}_{2}$ evoluído do solo pelo CBM determinado no solo (ANDERSON et al., 1993).

Os dados foram submetidos à análise de variância pelo teste $\mathrm{F}$ a $5 \%$ e $1 \%$ de probabilidade de erro. Interações significativas foram avaliadas e as médias comparadas pelo teste de Tukey $(p<0,05)$. 0 efeito dose foi avaliado por análise de regressão e modelos lineares simples $\left(\bar{Y}=\alpha+\beta_{1} X\right)$ e quadráticos $\left(\bar{Y}=\alpha+\beta_{1} X+\beta_{2} X^{2}\right)$ foram ajustados com base nos níveis de significância dos coeficientes em suas equações $(p<0,05)$ e no valor do seu coeficiente de determinação. As análises estatísticas foram realizadas com o software Sisvar ${ }^{\circledR}$ (FERREIRA, 2011). Gráficos de regressão e curvas foram criados usando o software SigmaPlot $^{\circledR}$ (Systat Software, Inc., Chicago, IL, USA).

\section{RESULTADOS E DISCUSSÃO}

Altura de planta obteve resultado significativo apenas para a variável inoculação. A altura da planta foi maior quando inoculado com Bradyrhizobium sp., para todas as doses estudadas (Figura 1A). A produção de massa seca da raiz foi maior na dose 0 e $800 \mathrm{~g} \mathrm{ha}^{-1}$ quando não receberam inoculação e as doses $400 \mathrm{e}$ 
$1200 \mathrm{~g} \mathrm{ha}^{-1}$ não diferiram ( $p>0,05$ ) (Figura 1B). Maior desenvolvimento em altura também foi observado em Canavalia ensiformis inoculada com Bradyrhizobium sp. (MIELKE et al., 2020). Os microrganismos podem ajudar no crescimento da planta através da excreção de substâncias promotoras de crescimento vegetal, como fitormônios (ácido indolacético, etileno), aminoácidos e substâncias reguladoras do metabolismo (THULER et al., 2003). A inoculação da crotálaria com Bradyrhizobium sp. não alterou o desenvolvimento da planta em relação à massa seca da raiz, o que pode estar relacionado a capacidade da planta em tolerar o sulfentrazone no solo sem que isso afete o acúmulo de fitomassa da planta fitorremediadora. Resultados semelhantes foram encontrados em trabalhos com mucuna-preta (Stizolobium aterrimum) e o herbicida tebuthiuron, tendo sido verificada fitotoxicidade menos acentuada e menor redução na altura das plantas, da biomassa seca da parte aérea e de raízes, em comparação com outras culturas (PIRES et al., 2003). Camargo et al. (2011) avaliaram a mucuna-anã (Stizolobium deeringianum) e mucuna-preta (S. aterrimum), inoculadas e não inoculadas com Rhizobium sp., ao herbicida atrazine. Os resultados encontrados para as variáveis altura, biomassa verde e seca para mucuna-preta não apresentaram diferença estatística entre os tratamentos com e sem inoculante, mostrando uma resistência natural à atrazine e a possibilidade de atuar como planta fitoremediadora.


Figura 1: Altura de plantas (A) e massa seca da raiz (B) de Crotalaria juncea, com e sem inoculação com Bradyrhizobium sp., cultivada em solo contaminado com diferentes doses de sulfentrazone. As barras verticais representam os desvios padrão $( \pm D P)$ das médias $(n=3)$. Barras seguidas pela mesma letra, na mesma dose, não diferiram significativamente de acordo com o teste de Tukey a $5 \%$.

O número e massa seca de nódulos foi significativo apenas para variável inoculação. O número de nódulos total foi maior quando realizada a inoculação com Bradyrhizobium sp., para a dose 0 e $1200 \mathrm{~g} \mathrm{ha}^{-1}$ não variando de modo significativo para as demais doses $(p>0,05)$ (Figura 2). A massa seca dos nódulos foi maior apenas para a dose $0 \mathrm{~g} \mathrm{ha}^{-1} \mathrm{com}$ a inoculação com Bradyrhizobium sp. As doses do herbicida foram prejudiciais para os microrganismos e consequentemente não tiveram resultados na formação dos nódulos, mostrando resultado apenas quando não se aplicou o herbicida. Embora a adição de bactérias biodegradativas a solos contaminados (biorremediação) seja comumente eficaz em facilitar a degradação dos contaminantes em um ambiente, a biorremediação bem-sucedida é muitas vezes mais difícil de alcançar. Isso ocorre porque mesmo que os microrganismos apresentem alta tolerância ao herbicida, o crescimento é geralmente reduzido significativamente, de modo que são incapazes de atingir massa suficiente para permitir 
que degradem eficientemente o herbicida em um período de tempo razoável (GLICK et al., 2010). Além disso, o efeito tóxico de alguns herbicidas foi observado logo após sua aplicação e algumas moléculas, como o paraquat, tiveram efeito duradouro de intoxicação para maioria dos microrganismos em estudo realizado por Adomako et al. (2016). Os autores observaram que o padrão de mudança pode variar como resultado de diferenças no período de exposição ao herbicida, a concentração do ingrediente ativo na formulação, tempo de exposição e fatores ambientais. Isso é apoiado pela visão de que a resposta microbiana aos herbicidas se manifesta de várias maneiras, dependendo de fatores, incluindo o próprio herbicida, populações de microrganismos inerentes, concentração de herbicida, tempo de exposição e características químicas e físicas do solo.
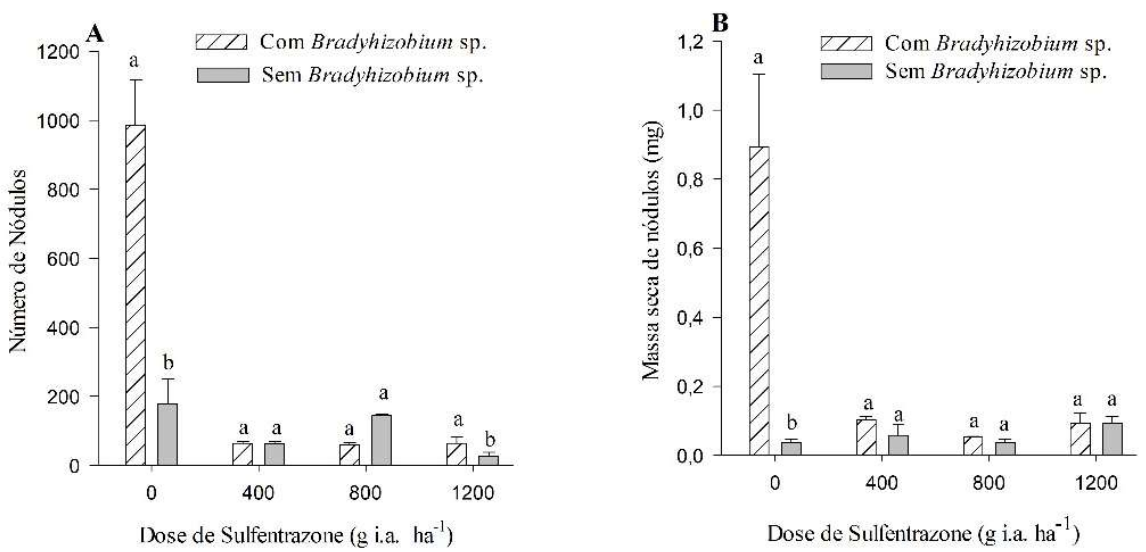

Figura 2: Número de nódulos (A) e Massa seca de nódulos (B) de Crotalaria juncea, associado ou não a Bradyrhizobium sp., cultivada em solo contaminado com diferentes doses de sulfentrazone. As barras verticais representam os desvios padrão $( \pm D P)$ das médias $(n=3)$. Barras seguidas pela mesma letra, na mesma dose, não diferiram significativamente de acordo com o teste de Tukey a $5 \%$.

A respiração microbiana foi significativa apenas para variável inoculação, sendo os dados submetidos ao teste de Tukey. A dose $0 \mathrm{~g} \mathrm{ha}^{-1}$ apresentou maior respiração quando não inoculado do que com inoculação (445,34 e 324,92 $\mu \mathrm{g} \mathrm{C}-\mathrm{CO}_{2} \mathrm{~g}^{-1}$, respectivamente). Sem inoculação, a dose de $400 \mathrm{~g} \mathrm{ha}^{-1}$ obteve maior respiração em comparação com inoculado (419,68 e 296,93 $\mu \mathrm{g} \mathrm{C}-\mathrm{CO}_{2} \mathrm{~g}^{-1}$, respectivamente). As doses de 800 e $1200 \mathrm{~g} \mathrm{ha}^{-1}$ não apresentaram diferença para inoculação $\left(379,75\right.$ e $338,12 \mu \mathrm{g} \mathrm{C}-\mathrm{CO}_{2} \mathrm{~g}^{-1}$, respectivamente) (Figura 3). O cultivo da C. juncea em simbiose com Bradyrhizobium sp. pode não ter favorecido a microbiota do solo durante o processo de remediação, sendo influenciado pela comunidade microbiana que se estabeleceu no solo durante o tempo de cultivo. Apesar do solo ter sido submetido ao processo de autoclavagem, a comunidade microbiana do solo pode se reestabelecer ao longo do tempo de cultivo da crotalária no solo. Esse fato pode estar relacionado à morte de microrganismos pelo processo de autoclavagem, o qual favoreceu a degradação dos compostos orgânicos usados como fonte de energia para os microrganismos que cresceram ao longo do período de cultivo (SOUZA et al., 2017). 0 estímulo à atividade microbiana, promovida pela liberação de substâncias radiculares, favorece a multiplicação de microrganismos que podem atuar degradando o herbicida no solo, caracterizando, em algumas plantas, como aptidão rizosférica na remediação desses compostos (MADALÃO et al., 2016).

O aumento proporcionado na frequência respiratória indica uma intensa atividade dos 
microrganismos e pode ser um indicativo da decomposição rápida de resíduos orgânicos (SANTOS et al., 2009). A degradação microbiana do sulfentrazone pode ser através de reações enzimáticas catabólicas, onde microrganismos do solo podem utilizar o herbicida como fonte de energia, ou através do co-metabolismo, que requer outra fonte de carbono e energia para sustentar o crescimento microbiano (BRUM et al., 2013; MELO et al., 2017).

Outro fator importante é o estádio em que as plantas foram manejadas/colhidas. O experimento foi conduzido até o pleno florescimento. Esta fase é caracterizada pela alta atividade metabólica das plantas e na qual há maior liberação de exsudados radiculares, contribuindo para maior número de microrganismos associados à rizosfera (AULAKH et al., 2001). Este fato pode ter tornado mais eficiente a degradação de sulfentrazone nos solos não inoculados. De forma semelhante, o cultivo de Stizolobium aterrimum até seu completo florescimento favoreceu o acúmulo de exsudatos na região das raízes, permitindo crescimento e desenvolvimento de maior número de grupos de microrganismos (SANTOS et al., 2007).

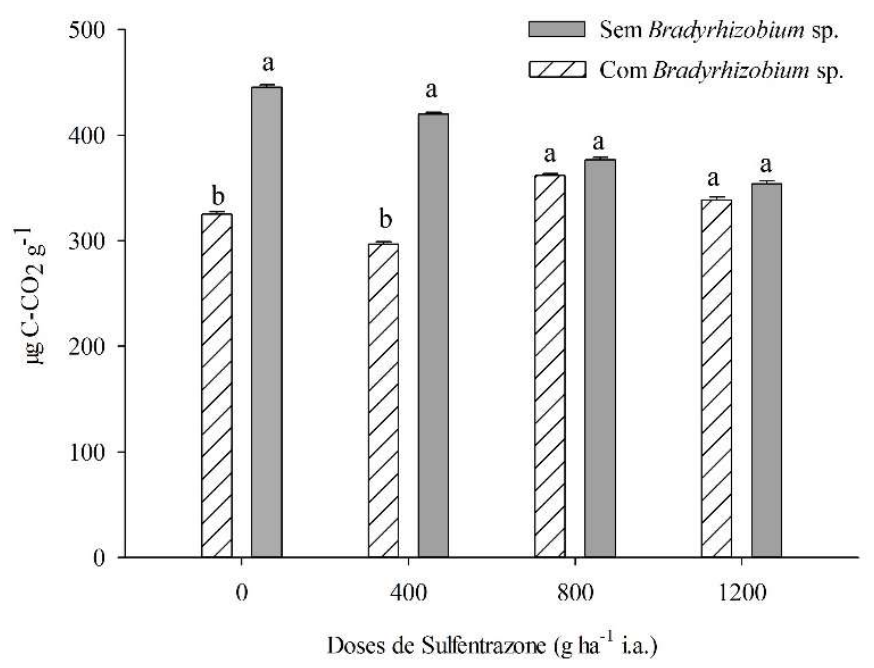

Figura 3: $\mathrm{C}-\mathrm{CO}_{2}$ total evoluído do solo cultivado com Crotalaria juncea, associado ou não a Bradyrhizobium sp., em função das doses de sulfentrazone. As barras verticais representam os desvios padrão $( \pm D P)$ das médias $(n=3)$. Barras seguidas pela mesma letra, na mesma dose, não diferiram significativamente de acordo com o teste de Tukey a $5 \%$.

O carbono da biomassa microbiana aumentou conforme aumentaram as doses (Figura 4A). cociente metabólico diminuiu conforme aumentaram as doses do sulfentrazone (Figura 4B). A respiração e cociente metabólico não foram afetados pela inoculação. A biomassa microbiana é considerada a fração viva da matéria orgânica do solo e um expressivo reservatório de nutrientes nos solos, atuando ativamente no processo de decomposição/mineralização de resíduos orgânicos (KASCHUK et al., 2010). Assim, a biomassa microbiana ativa, associada à crotalária, é de grande interesse, pois pode intensificar a descontaminação do solo com sulfentrazone no processo de biorremediação.

A taxa de respiração por unidade de biomassa microbiana, conhecida como quociente metabólico $\left(\mathrm{qCO}_{2}\right)$, foi aceito como um indicador sensível da resposta dos estresses ambientais sob a comunidade microbiana do solo (WARDLE et al., 1995). Altos valores de $\mathrm{qCO}_{2}$ são encontrados em locais submetidos a condições adversas, ou seja, em solos onde a biomassa microbiana oxida mais carbono para sua manutenção (LACERDA et al., 2013). Os baixos valores para $\mathrm{qCO}_{2}$, independente da inoculação com Bradyrhizobium sp., 
para 800 e $1200 \mathrm{~g} \mathrm{ha}^{-1}$ (Figura 4B), podem estar relacionados ao efeito não limitante do herbicida sobre a comunidade microbiana e possivelmente reflete um ambiente mais estável na rizosfera da crotalária. De forma semelhante, a aplicação do picloram não afetou o carbono da biomassa microbiana e o $\mathrm{qCO}_{2}$ nos solos rizosféricos de Panicum maximum e Urochloa. brizantha indicando que a rizosfera dessas espécies pode contribuir para a descontaminação dos solos tratados com picloram (SOUZA et al., 2017).

A respiração microbiana estável nas maiores doses do sulfentrazone (Figura 3) e maior carbono da biomassa e menor coeficiente metabólico (Figura 4) mostram que o potencial da degradação do herbicida na rizosfera da crotalária independe da aplicação do Bradyrhizobium sp. no solo. A comunidade microbiana em equilíbrio se torna mais eficiente em utilizar os recursos disponíveis e, consequentemente, maior quantidade de carbono pode ser incorporada aos tecidos microbianos (SANTOS et al., 2007). Assim, os resultados sugerem que a crotalária pode ser melhor beneficiada pela microbiota presente do solo, uma vez que a inoculação não promoveu benefícios na degradação microbiana do herbicida no solo e a respiração microbiana mostrou-se mais elevada sem o Bradyrhizobium sp.
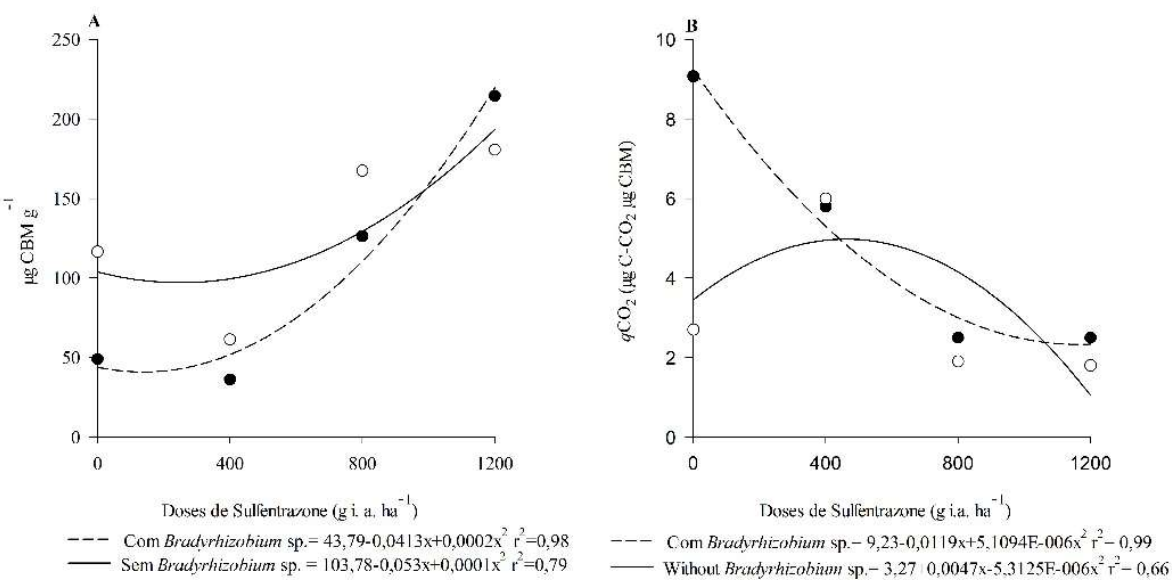

Figura 4: Carbono da biomassa microbiana (A) e Quociente metabólico (B) do solo contaminado com sulfentrazone, cultivado com Crotalaria juncea, associado ou não a Bradyrhizobium sp.

As variáveis analisadas nas plantas no bioensaio com milheto (Pennisetum glaucum) seguiram tendência de ajuste a um modelo quadrático, independente da inoculação (Figura 5). Não houve diferenças significativas para a variável inoculação $(p>0,05)$. À medida que aumentou a dose de sulfentrazone no solo à altura (Figura 5B) e a massa seca (Figura 5A) das plantas de milheto diminuíram, consequentemente, as injúrias (Figura 5C) na parte aérea aumentaram. Os resultados que indicam a eficiência de fitorremediação, nas doses $400 \mathrm{e} 800 \mathrm{~g} \mathrm{ha}^{-1}$, assemelharam-se muito e indicaram menores danos às plantas de milheto, mesmo sem o inoculante (Figura 5). Pela análise de regressão nota-se, entretanto, a tendência de melhores resultados para os tratamentos não inoculados. Isso demonstra o efeito do inoculante nas plantas de crotalária quanto à remediação do solo contaminado com sulfentrazone, possivelmente, pelo estímulo da atividade microbiana presente no solo, como mostrado nos resultados de respiração e biomassa microbiana (Figura 3 e 4).

Independentemente das limitações exibidas e das respostas à inoculação, os resultados para 
crotalária corroboram Madalão et al. (2012), que observaram um aumento na produção de biomassa quando C. juncea foi cultivada antes de $P$. glaucum, permitindo que este se desenvolvesse em um solo previamente contaminado com sulfentrazone, e também Belo et al. (2016), que indicaram que a fitorremediação como técnica de descontaminação de áreas onde persistem resíduos do herbicida sulfentrazone, utilizando as espécies vegetais $C$. ensiformis e $C$. juncea, pode possibilitar o início do cultivo de milheto nessas áreas em até 12 meses antes do que seria possível.
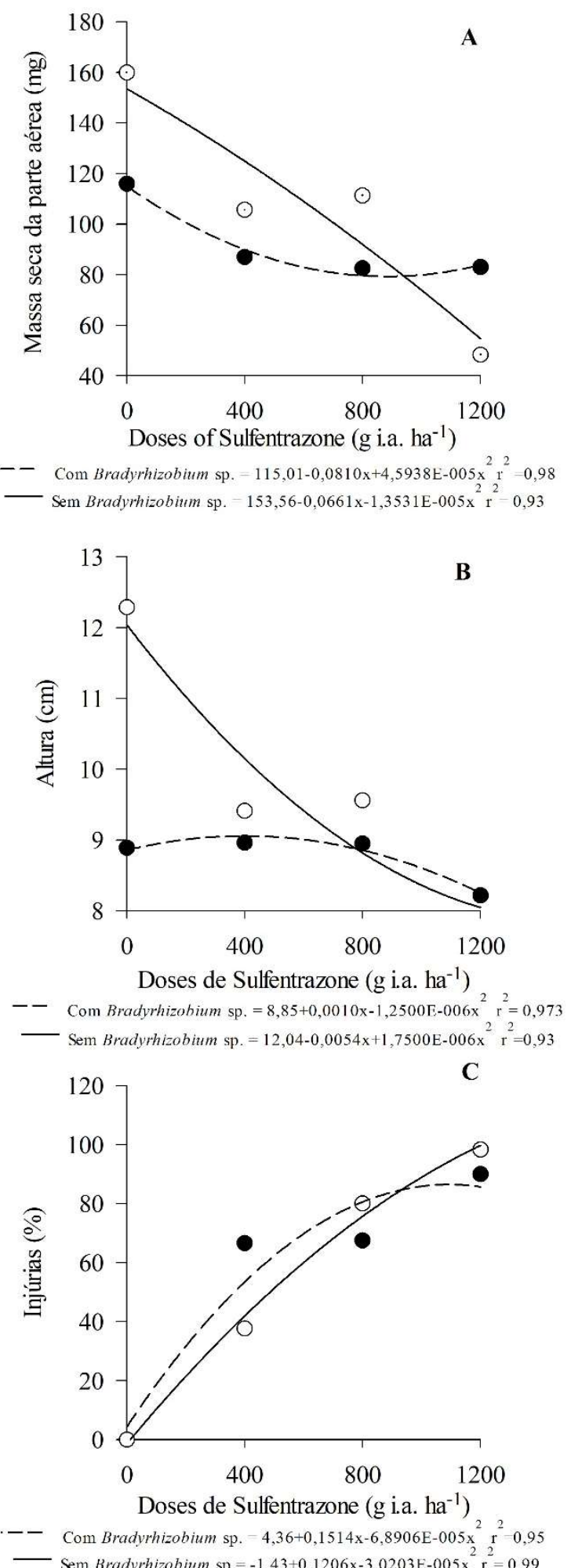

Figura 5: Massa seca da parte aérea (A), Altura de planta (B) e Injúrias visual (C) de Pennisetum glaucum cultivado em solo fitorremediado com Crotalaria juncea, associado ou não a Bradyrhizobium sp. 


\section{CONCLUSÕES}

A inoculação com Bradyrhizobium sp. proporcionou melhor desenvolvimento em altura da parte aérea das plantas de crolátaria, entretanto, o sistema radicular não foi influenciado pela adição dos microrganismos, mostrando maior benefício quando não inoculado.

Os indicadores biológicos do solo mostraram que a inoculação com Bradyrhizobium sp. influenciou de maneira negativa na atividade microbiana dos solos.

A crotalária apresentou boa tolerância aos níveis de sulfentrazone no solo, independentemente do inoculante. Doses mais elevadas do sulfentrazone apresentaram maior valor de biomassa microbiana e menor cociente metabólico, indicando maior atividade microbiana no solo, independente da inoculação.

Os resultados sugerem que a inoculação da crotalária com Bradyrhizobium sp. não mostrou benefício na eficácia da remediação do sulfentrazone.

AGRADECIMENTOS: Os autores agradecem ao Coordenação de Aperfeiçoamento de Pessoal de Nível Superior (CAPES) e Conselho Nacional de Desenvolvimento Científico e Tecnológico (CNPq) pela concessão de bolsas de pesquisa.

\section{REFERÊNCIAS}

ADOMAKO, M. O.; AKYEAMPONG, S.. Effect of commonly used herbicides on soil microbial population. Journal of Environmental Earth Science, v.6, n.1, p.30-38, 2016.

ANDERSON, T. H.; DOMSCH, K. H.. The metabolic quotient for $\mathrm{CO}_{2}\left(q \mathrm{CO}_{2}\right)$ as a specific activity parameter to assess the effects of environmental conditions, such as ph, on the microbial biomass of forest soils. Soil Biology and Biochemistry, v.25, n.3, p.393-395, 1993. DOI: https://doi.org/10.1016/0038-0717(93)90140-7

AULAKH, M. S.; WASSMANN, R.; BUENO, C.; RENNENBERG, $\mathrm{H}$.. Impact of root exudates of different cultivars and plant development stages of rice (Oryza sativa L.) on methane production in a paddy soil. Plant Soil, Amsterdã, v.230, p.7786, 2001. DOI: https://doi.org/10.1023/A:1004817212321

BELO, A. F.; PIRES, F. R.; BONOMO, R.; CARGNELUTTI FILHO, A.; TENIS, L. H. O.. Sulfentrazone phytoremediation under field conditions. Revista Caatinga, Mossoró, v.29, p.119-126, 2016. DOI: https://doi.org/10.1590/198321252016v29n114rc

BRUM, C. S.; FRANCO, A. A.; SCORZA JÚNIOR, R. P.. Degradação do herbicida sulfentrazone em dois solos de Mato Grosso do Sul. Revista Brasileira de Engenharia Agrícola e Ambiental, Campina Grande, v.17, n.5, p.558-564, 2013. DOI: https://doi.org/10.1590/s141543662013000500014

CAMARGO, D.; BISPO, K. L.; SENE, L.. Associação de Rhizobium sp. a duas leguminosas na tolerância à atrazina. Revista Ceres, Viçosa, v.58, n.4, p.425-431, 2011. DOI: https://doi.org/10.1590/S0034-737X2011000400004

COSTA, A. F.; SILVA, J. R. R.; SANTOS, R.; FARIAS, C. B. B.;
SARRUBO, L. A.; JORDÃO, R. C.; OLIVEIRA, R. A. D.. Obtenção de consórcio de microrganismos a partir de amostra de petróleo. Revista Ciências \& Tecnologia, v.1, p.1-7, 2007.

CURL, E. A.; RODRIGUEZ-KABANA, R.. Microbial interactions. In: WILKINSON, R. E.. Research Methods in Weed Science. Atlanta: Southern Weed Science Society, 1972. p.162-194.

DAN, H. A.; DAN, L. G. M.; BARROSO, A. L. L.; PROCÓPIO, S. O.; OLIVEIRA JUNIOR, R. S.; ASSIS, R. L.; SILVA, A. G.; FELDKIRCHER, C.. Effect of the residual activity of preemergent herbicides applied in soybean on pearl millet cultivated in succession. Planta Daninha, Viçosa, v.29, n.3, p.437-445, 2011. DOI: https://doi.org/10.1590/s010083582011000200022

EMBRAPA. Empresa Brasileira de Pesquisa Agropecuária. Sistema brasileiro de classificação de solos. 5 ed. Brasília: EMBRAPA, 2018.

FERRAÇO, M.; PIRES, F. R.; BELO, A. F.; CELIN FILHO, A.; BONOMO, R.. Efeito da densidade populacional de Canavalia ensiformis na fitorremediação de solo contaminado com sulfentrazone. Revista Ciência Agronômica, v.48, n.1, p.3240, 2017. DOI: https://doi.org/10.5935/1806$\underline{6690.20170004}$

FERREIRA, D. F.. Sisvar: a computer statistical analysis system. Ciência e Agrotecnologia, v.35, p.1039-1042, 2011. DOI: https://doi.org/10.1590/s1413-70542011000600001

GAGE, D. J.. Infection and invasion of roots by symbiotic, nitrogen-fixing rhizobia during nodulation of temperate legumes. Microbiology and Molecular Biology Reviews, v.68, n.2, p.280-300, 2004. DOI: https://doi.org/10.1128/MMBR.68.2.280-300.2004 
GLICK, B. R.. Using soil bacteria to facilitate phytoremediation. Biotechnology Advance, v.28, p.367-374, 2010. DOI:

https://doi.org/10.1016/j.biotechadv.2010.02.001

IMFELD, G.; VUILLEUMIER, S.. Measuring the effects of pesticides on bacterial communities in soil: A critical review. European Journal of Soil Biology, v.49, p.22-30, 2012. DOI: https://doi.org/10.1016/i.ejsobi.2011.11.010

ISLAM, K. R.; WEIL, R. R.. Microwave irradiation of soil for routine measurement of microbial biomass carbon. Biology and Fertility of Soils, v.27, p.408-416, 1998. DOI: https://doi.org/10.1007/s003740050451

MIELKE, K. C.; BERTUANI, R. R.; PIRES, F. R.; COTTA, A. J. B.; EGREJA FILHO, F. B.; MADALÃO, J. C.. Does Canavalia ensiformis inoculation with Bradyrhizobium sp. enhance phytoremediation of sulfentrazone-contaminated soil? Chemosphere, v.255, p.127033, 2020.

KANG, J. W.. Removing environmental organic pollutants with bioremediation and phytoremediation. Biotechnology Letters, v.36, p.1129-1139, 2014. DOI: https://doi.org/10.1007/s10529-014-1466-9

KASCHUK, G.; ALBERTON, O.; HUNGRIA, M.. Three decades of soil microbial biomass studies in Brazilian ecosystems: Lessons learned about soil quality and indications for improving sustainability. Soil Biology and Biochemistry, v.42, n.1, p.1-13, 2010. DOI:

https://doi.org/10.1016/j.soilbio.2009.08.020

LACERDA, K. A. P.; CORDEIRO, M. A. S.; VERGINASSI, A.; SALGADO, F. H. M.; PAULINO, H. B.; CARNEIRO, M. A. C.. Organic carbon, biomass and microbial activity in an Oxisol under different management systems. Revista de Ciências Agrarias - Amazon Journal of Agricultural and Environmental Sciences, Recife, v.56, n.3, p.249-254, 2013. DOI: https://doi.org/10.4322/rca.2013.036

KAWASAKI, A.; WATSON, E. R.; KERTESZ, M. A.. Indirect effects of polycyclic aromatic hydrocarbon contamination on microbial communities in legume and grass rhizospheres. Plant Soil, v.358, n.1, p.169-182, 2011.

MADALÃO, J. C.; PIRES, F. R.; CHAGAS, K.; CARGNELUTTI FILHO, A.; PROCÓPIO, S.. O Uso de leguminosas na fitorremediação de solo contaminado com sulfentrazone. Pesquisa Agropecuária Tropical, Goiânia, v.42, n.4, p.390396, 2012. DOI: https://doi.org/10.1590/S1983$\underline{40632012000400001}$

MADALÃO, J. C.; SILVA, A. A.; ORLANDO, W. A.; SARAIVA, D. T.; MELO, C. A. D.; D`ANTONINO, L.. The sulfentrazone interferes with microbial biomass and soil microbial activity. Revista de Ciências Agrarias - Amazon Journal of Agricultural and Environmental Sciences, Recife, v.59, p.5459, 2016. DOI: https://doi.org/10.4322/rca.2016

MARTINEZ, C. O.; SILVA, C. M. M. S.; FAY, E. F.; NUNES MAIA A. H.; ABAKERLI, R. B.; DURRANT, L. R.. Degradation of the herbicide sulfentrazone in a Brazilian Typic Hapludox soil. Soil Biology and Biochemistry, v.40, n.4, p.853-860, 2008. DOI: https://doi.org/10.1016/j.soilbio.2007.11.008
MELO, C. A. D.; PASSOS, A. B. R. J.; MADALÃO, J. C.; SILVA, D. V.; MASSENSSINI, A. M.; SILVA, A. A.; COSTA, M. D.; SOUZA, $M$. F.. Bioaugmentation as an associated technology for bioremediation of soil contaminated with sulfentrazone. Ecological Indicators, v.99, p.343-348, 2019. DOI: https://doi.org/10.1016/j.ecolind.2018.12.034

MONQUEIRO, P. A.; SILVA, P. V.; SILVA, H. A. C.; TABLAS, D. C.; ORZARI, I.. Leaching and persistence of sulfentrazone and imazapic. Planta Daninha, Viçosa, v.28, p.185-195, 2010. DOI: https://doi.org/10.1590/S0100-83582010000100022

OLIVEIRA, M. A.; PIRES, F. R.; FERRAÇO, M.; BELO, A. F.. The validation of an analytical method for sulfentrazone residue determination in soil using liquid chromatography and a comparison of chromatographic sensitivity to millet as a bioindicator species. Molecules, v.19, p.10982-10997, 2014. DOI: https://doi.org/10.3390/molecules190810982

PELCASTRE, M. I.; IBARRA, J. V.; NAVARRETE, A. M.; ROSAS, J. C.; SANDOVAL, O. A.. Bioremediation perspectives using autochthonous species of Trichoderma sp. for degradation of atrazine in agricultural soil from the Tulancingo Valley, Hidalgo, Mexico. Tropical and Subtropical Agroecosystems, v.16, n.2, p.265-276, 2013.

PIRES, F. R.; SOUZA, C.; SILVA, A.; PROCÓPIO, S.; FERREIRA, L.. Fitorremediação de solos contaminados com herbicidas. Planta Daninha, Viçosa, v.21, n.2, p.335-341.

RUFYIKIRI, G.; THIRY, Y.; DECLERCK, S.. Contribution of hyphae and roots to uranium uptake andtranslocation by arbuscular mycorrhizal carrot roots under root-organ culture conditions. New Phytologist, Oxon, v.158, n.2, p.391-399, 2003.

SANTOS, E. A.; SANTOS, J. B.; FERREIRA, L. R.; COSTA, M. D.; SILVA, A. A.. Phyto-stimulation by Stizolobium aterrimum as remediation of soil contaminated with trifloxysulfuronsodium. Planta Daninha, Viçosa, v.25, p.259-265, 2007. DOI: https://doi.org/https://doi.org/10.1590/S0100$\underline{83582007000200004}$

SANTOS, J. B.; FERREIRA, E. A.; FIALHO, C. M. T.; SANTOS, E. A.; GALON, L.; CONCEÇO, G.; AASIAZÚ, I.; SILVA, A. A.. Biodegradation of glyphosate in rhizospheric soil cultivated with Glycine max, Canavalia ensiformis and Stizolobium aterrimum. Planta Daninha, Viçosa, v.27, p.781-787, 2009. DOI: https://doi.org/10.1590/S0100-83582009000400016

SANTOS, N. M. C.; COSTA, V. A. M.; ARAÚJO, F. V.; ALENCAR B. T. B.; RIBEIRO, V. H. V.; OKUMURA, F.; SIMEONE, M. L. F.; SANTOS, J. B.. Phytoremediation of Brazilian tree species in soils contaminated by herbicides. Environmental Science and Pollution Research, v.25, p.27561-27568, 2018. DOI: https://doi.org/10.1007/s11356-018-2798-0

SANTOS, E. A.; CORREIA, N. M.; SILVA, J. R. M.; VELINI, E. D.; PASSOS, A. B. R. J.; DURIGAN, J. C.. Herbicide detection in groundwater in Córrego Rico-SP watershed. Planta Daninha, Viçosa, v.33, p.147-155, 2015. DOI: https://doi.org/10.1590/s0100-83582015000100017

SOUZA, W. M.; GUIMARÃES, F. A. R.; SOUZA, M. F.; SILVA, D. V.; MELO, C. A. D.. Rhizospheric activity of phytoremediation species in soil contaminated with picloram. Pesquisa Agropecuária Tropical, Goiânia, v.47, n.2, p.127-133, 2017. 
DOI: https://doi.org/10.1590/1983-40632016v4743289

STOTZKY, G.. Microbial respiration. In: BLACK, C. A.; EVANS, D. D.; ENSNUNGER, L. E.; VAUTE, J. L.; CLARK, F. E.. Methods of soil analysis: chemical and microbiological properties. Madison: American Society of Agronomy, 1965. p.15501572.

SHAW, L. J.; BURNS, R. G.. Enhanced mineralisation of [U-14C] 2,4-dichlorophenoxyacetic acid in thesoil from the rhizosphere of Trifolium pratense. Applied and Environmental Microbiology, v.70, n.8, p.4766-4774, 2004. DOI: https://doi.org/10.1128/AEM.70.8.4766-4774.2004

THULER, D. S.; FLOH, E. I. S.; HANDRO, W.; BARBOSA, H. R.. Beijerinckia derxii releases plant growth regulators and amino acids in synthetic media independent of nitrogenase activity. Journal of Applied Microbiology, v.95, n.4, p.799806, 2003. DOI: https://doi.org/10.1046/j.13652672.2003.02047.x
TRUU, J.; TRUU, M.; ESPENBERG, M.; NÕLVAK, H.; JUHANSON, J.. Phytoremediation and plant-assisted bioremediation in soil and treatment wetlands: a review. The Open Biotechnology Journal, v.9, n.1, p.85-92, 2015. DOI: https://doi.org/10.2174/1874070720150430E009

VANCE, E. D.; BROOKES, P. C.; JENKINSON, D. S.. An extraction method for measuring soil microbial biomass $C$. Soil Biology and Biochemistry, v.19, n.6, p.703-707, 1987. DOI: https://doi.org/10.1016/0038-0717/87)90052-6

WARDLE, D. A.; GHANI, A. A.. A critique of the microbial metabolic quotient $\left(q \mathrm{CO}_{2}\right)$ as a bioindicator of disturbance and ecosystem development. Soil Biology and Biochemistry, v.27, n.12, p.1601-1610, 1995. DOI: https://doi.org/10.1016/0038-0717(95)00093-T

ZAHRAN, H. H.. Rhizobium-legume symbiosis and nitrogen fixation under severe conditions and in anarid climate. Microbiology and Molecular Biology, v.63, p.968-989, 1999.

A CBPC - Companhia Brasileira de Produção Científica (CNPJ: 11.221.422/0001-03) detém os direitos materiais desta publicação. Os direitos referem-se à publicação do trabalho em qualquer parte do mundo, incluindo os direitos às renovações, expansões e disseminações da contribuição, bem como outros direitos subsidiários. Todos os trabalhos publicados eletronicamente poderão posteriormente ser publicados em coletâneas impressas sob coordenação da Sustenere Publishing, da Companhia Brasileira de Produção Científica e seus parceiros autorizados. Os (as) autores (as) preservam os direitos autorais, mas não têm permissão para a publicação da contribuição em outro meio, impresso ou digital, em português ou em tradução. 